\title{
Plant Regeneration from Shoot Apex-Derived Calluses of Marigold (Tagetes erecta L.)
}

\author{
María de Lourdes Miranda-Ham, ${ }^{1}$ Lizbeth A. Castro-Concha, \\ Elide Avilés-Berzunza, and Gregorio Godoy-Hernández \\ Unidad de Bioquímica y Biología Molecular de Plantas, Centro de \\ Investigación Cientifica de Yucatán, \\ Calle 43 \#130, Chuburná de Hidalgo, Mérida, Yucatán, 97200, México
}

Additional index words. shoot apex, indirect organogenesis, MSB medium

Marigold (Tagetes erecta $\mathrm{L}$.) is an annual plant, native of Mexico, that belongs to the Asteraceae (Compositae) family. Its flowers are commercially cultivated, harvested, and processed on an important industrial scale as a source of high-value pigments of the carotenoid family. Marigold flowers are the most concentrated common source of carotenoids, with lutein accounting for $85 \%$ of their total carotenoid content (Barzana et al., 2002). The meal made from the flowers is used as a supplement in the poultry industry (for coloring skin and egg yolk). Industrial sources have stated that the increasing awareness of toxicity of synthetic products has paved the way for the use of pigments from natural sources such as marigold (DelgadoVargas et al., 2000). Bioactive extracts of different Tagetes tissues also exhibit nematocidal, fungicidal, and insecticidal activity (Vanegas et al., 2002).

The development of suitable methods for in vitro regeneration is one of the main prerequisites for genetic improvement using biotechnologic means. Marigold plants have been in vitro regenerated from different sources, including immature unpollinated disc florets (Kothari and Chandra, 1984), leaf callus and suspension cultures (albeit with a loss of morphogenic potential as a result of subculturing) (Kothari and Chandra, 1986), hypocotyls (Belarmino et al., 1992), shoot tip proliferation from adult plants (Misra and Datta, 1999), and leaf segments (Misra and Datta, 2001; Vanegas et al., 2002). It is noteworthy to point out that, to our knowledge, there are no reports on regeneration through indirect organogenesis from shoot apex-derived calluses in Tagetes erecta.

Genetic transformation, either by particle bombardment or Agrobacterium infection, is a single cell event. Agrobacterium transformation requires tissue wounding previous to bacterial exposure. This may limit the number of transformed plants to those that regenerate only from the wounded area. Because methods using callus tissue do not

Received for publication 31 May 2006. Accepted for publication 9 July 2006.

${ }^{1}$ To whom reprint requests should be addressed; e-mailmirham@cicy.mx. require wounding, we believe that this protocol would increase the chances to obtain transformed plants after exposure of calluses by immersion to a bacterial suspension and further plantlet regeneration.

We report a consistent and reproducible protocol for the regeneration of plantlets from shoot apex-derived calluses of marigold without any loss of morphogenic potential resulting from subculturing. This protocol is based on the use of a modified Murashige and Skoog medium (MSB; Robert et al., 1987) containing different concentrations of benzyl adenine (BA) and indoleacetic acid (IAA). The highest mean number of shoots per explant was obtained with 70 $\mu \mathrm{M}$ BA and $10 \mu \mathrm{M}$ IAA 10 weeks after the culture's induction. When these shoots were excised and subcultured on MSB medium without plant growth regulators, they formed extensive root systems in vitro. Plantlets transferred to ex vitro conditions developed normally.

\section{Materials and Methods}

Marigold seeds (Tagetes erecta var. Orangeade Deep; PanAmerican Seed Co., Chicago) were surface-sterilized with a nonionic detergent for $5 \mathrm{~min}, 80 \%$ ethanol for 5 $\mathrm{min}$, and $2.5 \%$ sodium hypochlorite for 10 min and finally washed several times with sterile distilled water. The disinfested seeds were germinated on a plant growth regulators-free MS medium with a modified nitrogen source supplemented with 3\% sucrose and $0.23 \%$ gelrite, $\mathrm{pH} 5.8$. The modification consisted of a reduction of the total nitrogen content to $28 \mathrm{mM}$ with a $\mathrm{NO}_{3}{ }^{-} / \mathrm{NH}_{4}{ }^{+}$ratio of 4.6/1 (MSB medium; Robert et al., 1987). Fifteen seeds were placed in each glass jar $(10.5 \mathrm{~cm} \times 5 \mathrm{~cm})$ with $20 \mathrm{~mL}$ of MSB medium (Fig. 1A), and they were incubated at $25 \pm 2{ }^{\circ} \mathrm{C}$ under continuous light (40-50 $\left.\mu \mathrm{mol} \cdot \mathrm{m}^{-2} \cdot \mathrm{s}^{-1}\right)$. On germination, seedlings were transferred to fresh medium with the same composition and allowed to grow for 2 weeks. Shoot apexes (Fig. 1B, C) were excised and placed on the same MSB medium supplemented with different concentrations of IAA and BA as plant growth regulators and kept under the previously mentioned conditions. They were subcultured every 4 weeks and results were scored 10 weeks after the culture was initiated. Twenty explants were used for each treatment. The experiments were repeated independently at least three times with reproducible results.

The shoot forming capacity index (SFC) was calculated, according to Vanegas et al. (2002) with adaptations, to determine the efficiency of a given treatment, as follows:

$$
\mathrm{SFC}=(\mathrm{SPC}) \times(\mathrm{SFE}) / 100
$$

where SPC, the mean number of shoots per shoot apex-derived Calluses, represents the capacity of the explant to produce shoots, and SFE, the percentage of shoot forming explants, represents the response capacity of the tissue to the medium. The combination of these two index values allowed a better approximation to the optimal conditions for plant regeneration.

After 10 weeks, shoots with lengths $\geq 0.5$ $\mathrm{cm}$ were excised from shoot apex-derived calluses and transferred to glass jars $(10.5 \mathrm{~cm}$ $\times 5 \mathrm{~cm}$ ) with $40 \mathrm{~mL}$ of growth regulator-free MSB medium supplemented with 3\% sucrose to enhance plant growth and rooting. Plantlets were removed from the culture media, washed with tap water to remove any residual gelrite, and transferred to pots containing sterile soil and vermiculite (1:1). The pots were covered with transparent polyethylene bags and kept in a greenhouse at room temperature $\left(25\right.$ to $\left.30{ }^{\circ} \mathrm{C}\right)$ under natural illumination at a photon flux density of 310 $\mu \mathrm{mol} \cdot \mathrm{m}^{-2} \cdot \mathrm{s}^{-1}$. To reduce condensation, the bags had holes punched in them and after 1 week, they were removed. Three weeks later, the plants were transferred to the nursery, first under shading $\left(572 \mu \mathrm{mol} \cdot \mathrm{m}^{-2} \cdot \mathrm{s}^{-1}\right)$ and 3 weeks later exposed to open sunlight (1985 $\left.\mu \mathrm{mol} \cdot \mathrm{m}^{-2} \cdot \mathrm{s}^{-1}\right)$. The plants were watered daily and supplemented with Hoagland solution (Hoagland and Arnon, 1950) every 3 weeks.

\section{Results and Discussion}

Marigold plants have been regenerated from immature unpollinated disc florets (Kothari and Chandra, 1984), leaf calluses (Kothari and Chandra, 1986), and leaf explants (Vanegas et al., 2002) on MS medium using IAA and BA. To determine the best IAA and BA concentrations for shoot proliferation, several combinations were tested (Table 1). Shoot apexes cultured in small glass jars $(5 \mathrm{~cm} \times 3 \mathrm{~cm})$ with $9 \mathrm{~mL}$ of MSB medium, containing IAA and BA, gradually turned yellow and finally brown (Fig. 1D). However, 2 to 3 weeks later, small green calluses began to appear from the shoot apexes in all the growth regulators' tested combinations (Fig. 1D, E). Differentiation of shoots from shoot apex-derived calluses was achieved in several combinations after 10 weeks of culture (Table 1). Although shoot apex explants in media without growth regulators failed to form calluses, it was possible 

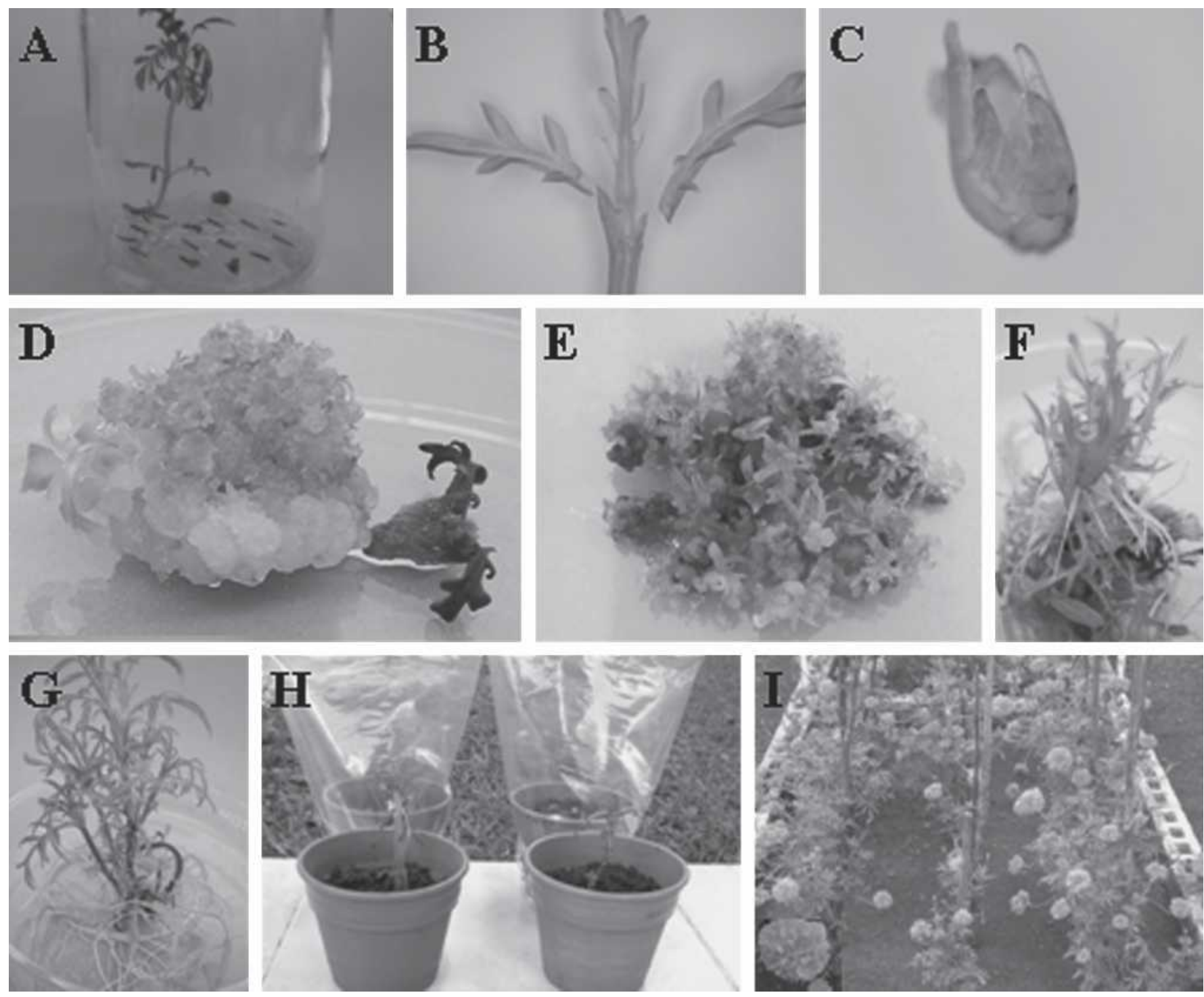

Fig. 1. In vitro shoot proliferation from shoot apex-derived calluses of marigold. (A) Seedling, (B) shoot apex, (C) apex, (D) shoots proliferating from calluses, (E) multiple shoots from shoot apex-derived calluses, (F) group of shoots, (G) rooting of plantlets, (H) acclimatization, and (I) flowering plants in the nursery.

Table 1. Effect of IAA and BA on shoot proliferation from shoot apex-derived calluses of Tagetes erecta. ${ }^{\text {. }}$

\begin{tabular}{|c|c|c|c|c|}
\hline $\begin{array}{l}\text { Treatment MSB + growth } \\
\text { regulators }(\mu \mathrm{M})\end{array}$ & Shoot/explant & Shoot size & $\begin{array}{l}\text { Percent explant } \\
\text { response }\end{array}$ & $\begin{array}{l}\text { Shoot forming } \\
\text { capacity }\end{array}$ \\
\hline $\mathrm{IAA}(10)+\mathrm{BA}(30)$ & $34.0 \pm 11.0 \mathrm{~b}$ & Between 0.5 and $1.5 \mathrm{~cm}$; multiple shoots $(<0.5 \mathrm{~cm})$ & 70 & 23.8 \\
\hline $\mathrm{IAA}(30)+\mathrm{BA}(30)$ & $22.0 \pm 5.0 \mathrm{ab}$ & Between 0.5 and $1.7 \mathrm{~cm}$; multiple shoots $(<0.5 \mathrm{~cm})$ & 70 & 15.4 \\
\hline $\mathrm{IAA}(30)+\mathrm{BA}(50)$ & $66.0 \pm 10.3 \mathrm{c}$ & Between 0.5 and $1.1 \mathrm{~cm}$; multiple shoots $(<0.5 \mathrm{~cm})$ & 40 & 26.4 \\
\hline $\mathrm{IAA}(10)+\mathrm{BA}(70)$ & $66.0 \pm 12.0 \mathrm{c}$ & Between 0.5 and $1.3 \mathrm{~cm}$; multiple shoots $(<0.5 \mathrm{~cm})$ & 70 & 46.2 \\
\hline $\mathrm{IAA}(30)+\mathrm{BA}(70)$ & $8.0 \pm 3.0 \mathrm{a}$ & Between 0.5 and $1.3 \mathrm{~cm}$; multiple shoots $(<0.5 \mathrm{~cm})$ & 40 & 3.2 \\
\hline
\end{tabular}

Means followed by the same letter in the shoot/explant column do not differ statistically at $P=0.05$ according to Tukey's test (SAS program).

${ }^{\mathrm{z}}$ Data presented are the mean number $( \pm \mathrm{SD})$ from 20 explants for each treatment recorded after 10 weeks of the culture's initiation.

MSB, Murashige and Skoog medium; IAA, indoleacetic acid; BA, benzyl adenine.

to regenerate marigold plants by direct organogenesis (Table 1). Misra and Datta (1999) reported the in vitro propagation of marigold through shoot tip proliferation using $22.19 \mu \mathrm{M}$ BA for $7 \mathrm{~d}$ followed by their transfer to a 50-times lower BA concentration $(0.44 \mu \mathrm{M})$. They stated that the use of $0.44 \mu \mathrm{M}$ BA proved to be the best for the induction of shoot buds among all the plant growth regulators tested.
The best combination for shoot proliferation from shoot apex-derived calluses based on the calculated SFC index (46.2) was $10 \mu \mathrm{M}$ IAA, $70 \mu \mathrm{M}$ BA (Table 1). In contrast, Vanegas et al. (2002) reported a SFC index of 1.39 using leaf explants in a media containing 17.1 $\mu \mathrm{M}$ IAA, 13.3 $\mu \mathrm{M}$ BA. Differences in genotype or in the nitrogen content in the media may account for the observed results.
Shoot vitrification in Tagetes erecta has been associated to the use of high BA concentrations in the culture medium. This has been reversed by diminishing the BA content along with the addition of silver nitrate (an inhibitor of ethylene biosynthesis) (Misra and Datta, 2001). Under our culture conditions (70 $\mu \mathrm{M}$ BA), shoots presented a slight vitrified aspect (Figs. 1D-F), but this negative feature was spontaneously overcome when 
shoots were transferred to the rooting medium (MSB medium without growth regulators; Fig. 1G).

When the differentiated shoots, along with the shoot apex-derived calluses, were kept in the same regeneration medium, they continued growing, rooting, and proliferating. To accelerate the rooting process, shoots were transferred to MSB medium without plant growth regulators for 3 to 4 weeks (Fig. 1G). When plantlets were $4 \mathrm{~cm}$ tall, they were transferred to pots with sterile soil and vermiculite (1:1) for acclimatization, as previously described (Fig. 1H). Misra and Datta (1999) reported that when shoots had reached up to a height of 3 to $4 \mathrm{~cm}$, they were rooted and that $100 \%$ rooting was achieved within $7 \mathrm{~d}$ of incubation in MS medium containing $0.05 \mathrm{mg} / \mathrm{L}$ NAA. Although rooting was achieved with any of the auxins they used, callusing, which preceded root formation, was undesirable for successful transplantation of the in vitro regenerated plantlets to soil. In our conditions, the frequency of survival was higher than $80 \%$ when rooting was performed in the absence of growth regulators. Khotari and Chandra (1984) reported that when 20 plants were transferred to soil, none of them developed to mature plants. In contrast, Misra and Datta (2001) reported a similar survival frequency (up to $75 \%$ ), whereas Vanegas et al. (2002) reported a $100 \%$ survival. It should be emphasized that all the acclimatized plants completed their life cycle normally, producing flowers and seeds in the nursery (Fig. 1I). Furthermore, no morphologic abnormalities were observed.

Medium composition is one of the key factors affecting morphogenesis in cells grown in vitro. Previous studies on differentiation have demonstrated the importance of the nitrogen source and the effects of the $\mathrm{NO}_{3}{ }^{-} / \mathrm{NH}_{4}{ }^{+}$ratio in the culture medium (Robert et al., 1987). It should be noted that MSB medium, when compared with MS medium, is characterized by a lowered total level of inorganic nitrogen but an increased $\mathrm{NO}_{3}{ }^{-} / \mathrm{NH}_{4}{ }^{+}$ratio. The molar ratio in the MSB media was $\approx 4.6 / 1$ and total level of inorganic nitrogen $28 \mathrm{~mm}$, whereas in MS medium, the molar ratio is $2 / 1$ and total level of inorganic nitrogen $60 \mathrm{~mm}$. In this way, the amount of available nitrogen appears to be less important for organogenesis than the nitrate/ammonium ratio, as has been found for other species such as Agave fourcroydes (Robert et al., 1987).

A two-stage method for in vitro regeneration, consisting of: 1) callus induction, maintenance, and shoot induction on the same MSB medium with subcultures every 4 weeks; and 2) rooting on MSB medium without growth regulators, is proposed for the in vitro propagation of marigold plants through culture of shoot apex-derived calluses in the present report.

One of the main advantages of this method is that the loss of the morphogenetic potential of calluses, resulting from an excessive number of subcultures, can be avoided. Regarding its use in the establishment of a stable protocol of genetic transformation, it must be pointed out that there could be a real higher probability that shoots may be derived from transformed cells. When using other explants such as leaf primordium, shoot tips, hypocotyls, radicles, and so on, for the same purpose, there are physical barriers (epidermal protection) to overcome for the transformation through Agrobacterium tumefaciens, hence the extensive use of wounding in such protocols (Godoy-Hernández et al., 2006). This method is being currently used to establish the stable genetic transformation protocol using the conditions previously reported for Agrobacterium-mediated transient transformation of marigold with GUS reporter gene (Godoy-Hernández et al., 2006) with encouraging results.

\section{Literature Cited}

Barzana, E., D. Rubio, R.I. Santamaría, O. GarcíaCorrea, F. García, V.E. Ridaura Sanz, and A. López-Mungía. 2002. Enzyme-mediated solvent extraction of carotenoids from marigold flowers (Tagetes erecta). J. Agr Food Chem. 50:4491-4496.

Belarmino, M.M., T. Abe, and T. Sasahara. 1992. Callus induction and plant regeneration in African marigold (Tagetes erecta L.). Ikushuga $\mathrm{Ku}$ Zasshi. 42:835-841.

Delgado-Vargas, F., A.R. Jiménez, and O. ParedesLópez. 2000. Natural pigments: carotenoids, anthocyanins, and betalains-Characteristics, biosynthesis, processing, and stability. Crit. Rev. Food Sci. Nutr. 40:173-289.

Godoy-Hernández, G., E. Avilés-Berzunza, L. Castro-Concha, and M.L. Miranda-Ham. 2006. Agrobacterium-mediated transient transformation of marigold (Tagetes erecta L.). Plant Cell Tiss. Org. 84:365-368.

Hoagland, D.R. and D.I. Arnon. 1950. The waterculture method for growing plants without soil Univ. of California Agric. Expt. Sta. Circ. 347. Univ. of California, Berkeley, Calif.

Kothari, S.L. and N. Chandra. 1984. Plant regeneration from cultured disc florets of Tagetes erecta L. J. Plant Physiol. 117:105-108.

Kothari, S.L. and N. Chandra. 1986. Plant regeneration in callus and suspension cultures of Tagetes erecta L. J. Plant Physiol. 122: 235-241.

Misra, P. and S.K. Datta. 1999. In vitro propagation of white marigold (Tagetes erecta L.) through shoot tip proliferation. Curr. Sci. 77:1141-1144.

Misra, P. and S.K. Datta. 2001. Direct differentiation of shoot buds in leaf segments of white marigold (Tagetes erecta L.). In Vitro Dev. Biol. Plant. 37:466-470.

Robert, M.L., J.L. Herrera, F. Contreras, and K.N. Scorer. 1987. In vitro propagation of Agave fourcroydes Lem. (Henequen). Plant Cell Tiss. Org. 8:37-48

Vanegas, P.E., A. Cruz-Hernández, M.E. Valverde, and O. Paredes-López. 2002. Plant regeneration via organogenesis in marigold. Plant Cell Tiss. Org. 69:279-283. 